\title{
Effects of Azelnidipine plus OlmesaRTAn versus amlodipine plus olmesartan on central blood pressure and left ventricular mass index: the AORTA study
}

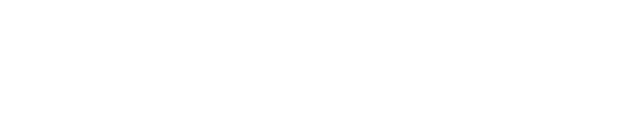

\author{
Takeshi Takami' \\ Yoshihiko Saito ${ }^{2}$ \\ 'Department of Internal Medicine, \\ Clinic Jingumae, Kashihara, Japan; \\ ${ }^{2}$ First Department of Internal \\ Medicine, Nara Medical University, \\ Kashihara, Japan
}

\begin{abstract}
Purpose: The aim of this study was to compare the effects of olmesartan combined with either azelnidipine or amlodipine on central blood pressure (CBP) and left ventricular mass index (LVMI) in hypertensive patients.

Patient and methods: Patients with brachial systolic BP $\geq 140 \mathrm{mmHg}$ and/or diastolic BP $\geq 90 \mathrm{mmHg}$ received olmesartan monotherapy (20 mg daily) for 12 weeks. The patients were then randomly assigned to fixed-dose add-on therapy with azelnidipine (16 mg daily) or amlodipine ( $5 \mathrm{mg}$ daily) (25 patients/group) for a further 24 weeks. CBP and LVMI were measured at baseline and at the end of the study.

Results: Baseline characteristics were similar in both groups. The decrease in brachial BP was similar in both groups. CBP and LVMI decreased significantly in both groups (both, $P<0.001$ ). However, the decreases in CBP and LVMI were significantly greater with olmesartan/ azelnidipine than with olmesartan/amlodipine (CBP, $P<0.001$; LVMI, $P=0.002$ ).

Conclusions: These findings indicate that olmesartan/azelnidipine had greater effects on CBP and LVMI than did olmesartan/amlodipine, even though the reduction in brachial BP was similar in both groups. These differential effects on CBP and LVMI may have important implications for cardiovascular risk reduction.
\end{abstract}

Keywords: central blood pressure, left ventricular mass index, augmentation index, brachial-ankle pulse wave velocity, olmesartan/azelnidipine

\section{Introduction}

When blood pressure (BP) control is inadequate with a single antihypertensive drug, the use of two or three drugs in combination is often necessary to achieve the target blood pressure. Combination therapy with an angiotensin II receptor blocker (ARB) and a diuretic or an ARB plus a calcium channel blocker (CCB) are recommended in the current Japanese Society for Hypertension guidelines. ${ }^{1}$ Several studies, including the ASCOT-CAFE ${ }^{2}$ and the Strong Heart Study, ${ }^{3}$ have emphasized the importance of targeting central blood pressure (CBP) rather than brachial BP in terms of cardiovascular disease outcomes. For example, the ASCOT-CAFÉ study compared the efficacy of amlodipine with that of atenolol, a $\beta$-blocker, and showed that both regimens had very different effects on central aortic pressures and hemodynamics despite similar effects on brachial BP. ${ }^{2}$ Meanwhile, in the Strong Heart Study, ${ }^{3}$ central pulse pressure was more strongly related to vascular hypertrophy, extent of atherosclerosis, and cardiovascular events than was brachial BP.
Correspondence: Takeshi Takami Department of Internal Medicine, Clinic Jingumae, 5-4-4I Kashihara Naizencho, Kashihara, Nara 634-0804, Japan

Tel +8I 744238568

Fax +8I 744236818

Email takami66@m5.kcn.ne.jp 
Several studies have also shown that an ARB in combination with a $\mathrm{CCB}$ significantly improves $\mathrm{CBP} .{ }^{4,5}$ Notably, however, the influence of CCBs on BP cannot be explained as a class effect because of differences in the effects of azelnidipine and amlodipine. ${ }^{6-8}$

Unfortunately, unlike amlodipine, few studies have examined the effects of azelnidipine on CBP. Moreover, to our knowledge, no studies of combination therapy have been conducted in Japan. In this context, we conducted the current study (Azelnidipine plus OlmesaRTAn versus amlodipine plus olmesartan; AORTA study) to determine the effects of adding either azelnidipine or amlodipine to ongoing olmesartan in patients with inadequate BP control on a standard dose of olmesartan.

\section{Methods}

\section{Patients}

Patients aged 36-75 years were recruited from among outpatients of the Department of Internal Medicine at Clinic Jingumae (Kashihara, Japan) between March 2007 and October 2008. We initially enrolled consecutive hypertensive patients with or without current treatment who agreed to participate in this study. Hypertension was defined as clinic-measured systolic BP (SBP) $\geq 140 \mathrm{mmHg}$ and/or diastolic $\mathrm{BP}(\mathrm{DBP}) \geq 90 \mathrm{mmHg}$ on two different occasions or by a previous diagnosis of hypertension with current antihypertensive therapy. Patients were excluded if they had secondary hypertension, arrhythmia, current treatment for congestive heart failure, a history of stroke or coronary artery disease, clinically significant valvular heart disease, renal insufficiency (serum creatinine $\geq 2 \mathrm{mg} / \mathrm{dL}$ ), mental disorders, severe noncardiovascular disease (eg, cancer or liver cirrhosis), or chronic inflammatory disease. Patients who were already being treated with olmesartan were also excluded. Written informed consent was obtained from all of the patients participating in the study. The study protocol was approved by the Institutional Ethical Committee.

\section{Study design}

The AORTA study was a 24-week, prospective, randomized, open-label parallel-group study comprising a 12-week run-in period followed by a 24-week randomized treatment period. The design of the study is summarized in Figure 1. Patients who were already being treated with antihypertensive drugs switched their current antihypertensive medications to olmesartan monotherapy. If the clinic SBP exceeded $200 \mathrm{mmHg}$ and/or DBP exceeded $115 \mathrm{mmHg}$ at any time during the run-in period, the patient was withdrawn from the study.

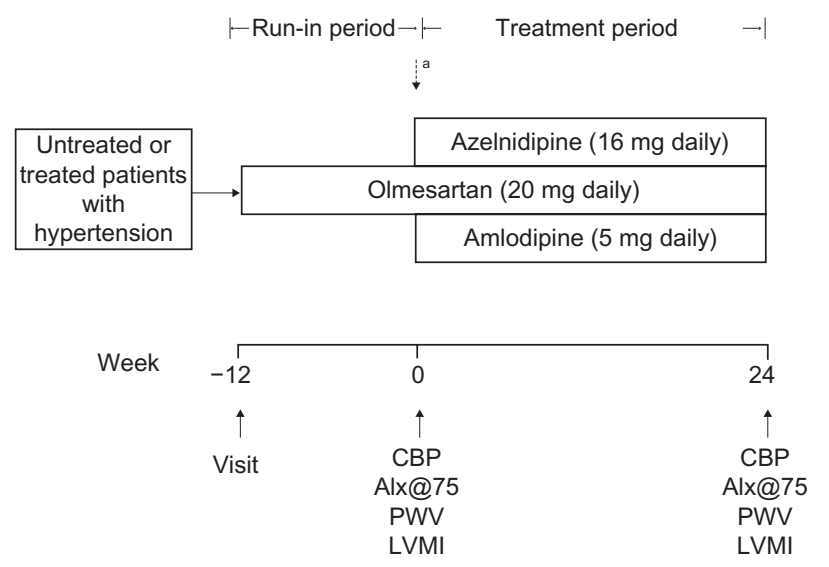

Figure I Study protocol.

Note: aPatients were randomized at the end of the run-in period if clinic-measured SBP was $\geq 140 \mathrm{mmHg}$ and/or DBP was $\geq 90 \mathrm{mmHg}$.

Abbreviations: SBP, systolic blood pressure; DBP, diastolic blood pressure; CBP, central blood pressure; Alx@75, normalized augmentation index; PWV, pulse wave velocity; LVMI, left ventricular mass index.

Both regimens were given at fixed doses for 24 weeks; dose titration was not permitted. The patients were instructed to take their medications after breakfast and were not permitted to use any antihypertensive drugs other than the study drugs. Other drugs that had the potential to interfere with the safety or efficacy of the study drugs were also not permitted. Brachial BP, heart rate (HR), CBP, normalized augmentation index (AIx@75), brachial-ankle pulse wave velocity (baPWV), and left ventricular mass index (LVMI) were measured at baseline and at the end of the 24-week treatment phase.

\section{Measurement of CBP, Alx@75, and baPWV}

The pulse pressure waveform of the radial artery was recorded using an automated tonometry system (HEM9000AI; Omron Healthcare, Kyoto, Japan) with the patient in the sitting position after resting for $\geq 5$ minutes. The waveform was automatically calibrated using the built-in oscillometric brachial sphygmomanometer, and the peak and trough of the radial pressure wave were adjusted to brachial SBP and DBP, respectively. An algorithm programmed into the HEM-9000AI system performed automatic online detection of the second peak (late systolic inflection) based on the second maxima of the fourth derivative of the radial pressure waveform to determine the radial AI as well as the late or second SBP (SBP2). This algorithm is described in more detail elsewhere. ${ }^{9}$ The height of the second peak corresponds to the SBP2 value obtained by the HEM-9000AI. SBP2 is very close to invasively recorded aortic $\mathrm{CBP}^{10}$ and was used 
as an estimate of CBP. CBP determined by the HEM9000-AI was reported to be comparable with CBP estimated using a generalized aorta-radial transfer function. ${ }^{11,12}$ The AI was calculated using the formula (SBP2 - DBP)/(the first peak $\mathrm{SBP}-\mathrm{DBP}) \times 100$. Because AI is influenced by the HR, it was normalized for an HR of 75 bpm (AIx@75) as proposed by Wilkinson et al. ${ }^{13}$ baPWV was also used to assess arterial stiffness. baPWV was performed as described previously. ${ }^{14}$ Briefly, baPWV was determined from the pulse waveforms recorded from both forearms and both ankles using the formula PWV (Omron Healthcare). This parameter was measured in patients who had been lying in the supine position for at least 5 minutes. baPWV measurements were repeated twice to confirm reproducibility, and the deviation between measurements was within $5 \%$. The mean value on the right side was used as the baPWV value in each patient.

\section{Measurement of LVMI}

M-mode echocardiography was performed under 2-dimensional guidance using a Vivid S6 ultrasound system (GE Healthcare, Milwaukee, WI) with a 3-MHz transducer. LVMI was measured by standard M-mode echocardiography and determined using the formula reported by Devereux et al. ${ }^{15}$

\section{Statistical analysis}

Data are shown as means $\pm \mathrm{SD}$. Differences between the two groups at baseline were analyzed using unpaired $t$ tests for continuous variables and $\chi^{2}$ tests for categorical variables. Paired $t$ tests were used for within-group comparisons while unpaired $t$ tests were used for between-group comparisons. Values of $P<0.05$ (2-sided) were considered statistically significant. SAS software (v 8.2; SAS Institute Inc, Cary, NC) was used for all analyses.

\section{Results}

\section{Baseline characteristics}

During the run-in period, 95 patients received $20 \mathrm{mg}$ olmesartan monotherapy once daily. At the end of the run-in period, 43 patients discontinued because they did not meet the BP criteria. Thus, 52 patients with a clinic $\mathrm{SBP} \geq 140 \mathrm{mmHg}$ and/or DPB $\geq 90 \mathrm{mmHg}$ were eligible and were randomized to receive add-on azelnidipine (16 mg daily) or amlodipine (5 mg daily) to ongoing olmesartan. Patients were randomized using the permuted block method. Of the 26 patients randomized to the olmesartan/azelnidipine regimen, one was excluded after missing the final assessment visit. Of the 26 patients randomized to the olmesartan/ amlodipine regimen, one was excluded after missing the final assessment visit. Thus, the population included 25 patients assigned to each regimen. The characteristics of the patients at the start of the randomized phase of the study (ie, baseline) are shown in Table 1. The baseline BP and other hemodynamic parameters were similar in both groups.

\section{Changes in brachial BP, CBP, and HR}

Brachial BP and CBP decreased significantly in both treatment groups (all, $P<0.001$; Table 2). However, the decrease in CBP was significantly greater in the olmesartan/ azelnidipine group than in the olmesartan/amlodipine group $(-14.0 \pm 4.3 \mathrm{vs}-8.3 \pm 3.7 \mathrm{mmHg}$, respectively, $P<0.001$ Figure 2A), whereas the decrease in brachial BP was similar in both groups. The magnitude of the decrease in HR was significantly greater in the olmesartan/azelnidipine group than in the olmesartan/amlodipine group $(-4.4 \pm 4.3 \mathrm{vs}$ $1.1 \pm 3.3 \mathrm{bpm}$, respectively, $P<0.001$; Figure $2 \mathrm{~B}$ ).

\section{Changes in Alx@75}

The AIx@75 decreased significantly in both treatment groups between baseline and endpoint (both $P<0.001$; Table 2). The magnitude of the decrease in AIx@75 was significantly greater in the olmesartan/azelnidipine group than in the olmesartan/amlodipine group $(-8.4 \pm 5.4$ vs $-4.3 \pm 3.7 \%$, respectively, $P<0.001$; Figure $2 \mathrm{C}$ ).

Table I Baseline characteristics

\begin{tabular}{llll}
\hline & $\begin{array}{l}\text { Olmesartan/ } \\
\text { Azelnidipine } \\
(\mathbf{n}=\mathbf{2 5})\end{array}$ & $\begin{array}{l}\text { Olmesartan/ } \\
\text { Amlodipine } \\
(\mathbf{n}=\mathbf{2 5})\end{array}$ & P-value \\
\hline Age (years) & $65.8 \pm 4.1$ & $67.5 \pm 4.6$ & 0.17 \\
Sex (male, \%) & $17(68 \%)$ & $18(72 \%)$ & 0.75 \\
BMI $\left(\mathrm{kg} / \mathrm{m}^{2}\right)$ & $25.8 \pm 1.0$ & $25.8 \pm 1.3$ & 0.97 \\
SBP $(\mathrm{mmHg})$ & $151.8 \pm 5.6$ & $151.6 \pm 4.4$ & 0.89 \\
DBP $(\mathrm{mmHg})$ & $85.8 \pm 5.2$ & $86.2 \pm 4.7$ & 0.75 \\
CBP $(\mathrm{mmHg})$ & $147.3 \pm 5.4$ & $146.3 \pm 3.5$ & 0.46 \\
HR $(\mathrm{bpm})$ & $73.5 \pm 5.8$ & $73.7 \pm 7.0$ & 0.93 \\
Alx@75 $(\%)$ & $91.9 \pm 4.5$ & $92.7 \pm 3.3$ & 0.48 \\
baPWV $(\mathrm{cm} / \mathrm{sec})$ & $1895.9 \pm 201.0$ & $1848.4 \pm 174.0$ & 0.38 \\
LVMI $\left(\mathrm{g} / \mathrm{m}^{2}\right)$ & $122.8 \pm 3.0$ & $122.8 \pm 2.8$ & 0.96 \\
eGFR $\left(\mathrm{mL} / \mathrm{min} / \mathrm{l} .73 \mathrm{~m}^{2}\right)$ & $66.4 \pm 4.1$ & $66.4 \pm 4.5$ & 0.97 \\
HbAIc $(\%)$ & $5.8 \pm 1.2$ & $5.7 \pm 1.1$ & 0.92 \\
LDL-C $(\mathrm{mg} / \mathrm{dL})$ & $110.3 \pm 16.5$ & $112.0 \pm 12.3$ & 0.67 \\
HDL-C $(\mathrm{mg} / \mathrm{dL})$ & $55.9 \pm 9.0$ & $55.4 \pm 8.3$ & 0.83 \\
TG $(\mathrm{mg} / \mathrm{dL})$ & $152.7 \pm 75.4$ & $170.4 \pm 61.9$ & 0.37 \\
UA $(\mathrm{mg} / \mathrm{dL})$ & $5.4 \pm 1.0$ & $5.6 \pm 0.8$ & 0.53 \\
\hline Not & &
\end{tabular}

Note: Values are means \pm standard deviation or $\mathrm{n}(\%)$.

Abbreviations: BMI, body mass index; SBP, systolic blood pressure; DBP, diastolic blood pressure; CBP, central blood pressure; HR, heart rate; Alx@75, normalized augmentation index; baPWV, brachial-ankle pulse wave velocity; LVMI, left ventricular mass index; eGFR, estimated glomerular filtration rate; LDL-C, lowdensity lipoprotein cholesterol; HDL, high-density lipoprotein cholesterol; TG, triglyceride; UA, uric acid. 


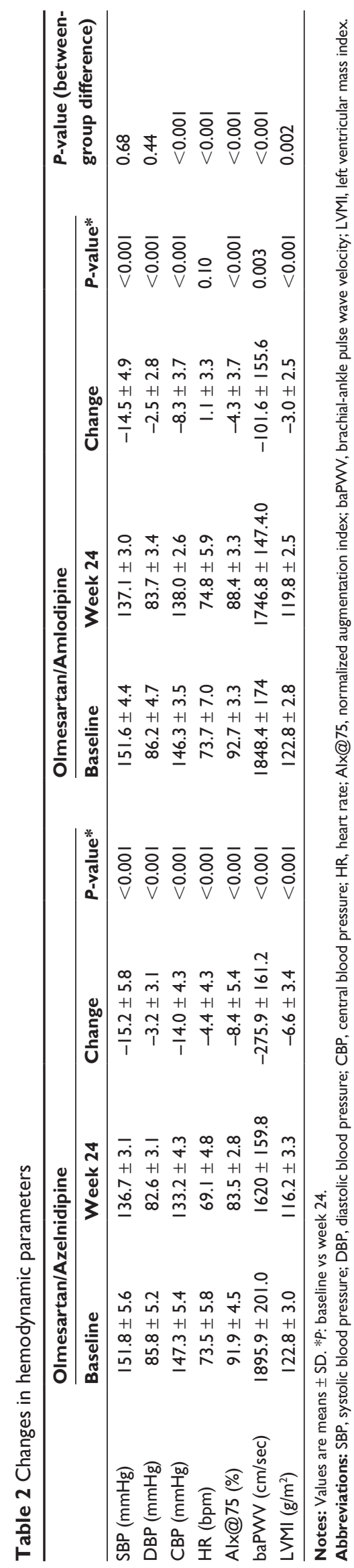

\section{Changes in baPWV}

baPWV decreased significantly in both treatment groups between baseline and endpoint (olmesartan/azelnidipine, $P<0.001$; olmesartan/amlodipine, $P=0.003$; Table 2). The magnitude of the decrease in baPWV was significantly greater
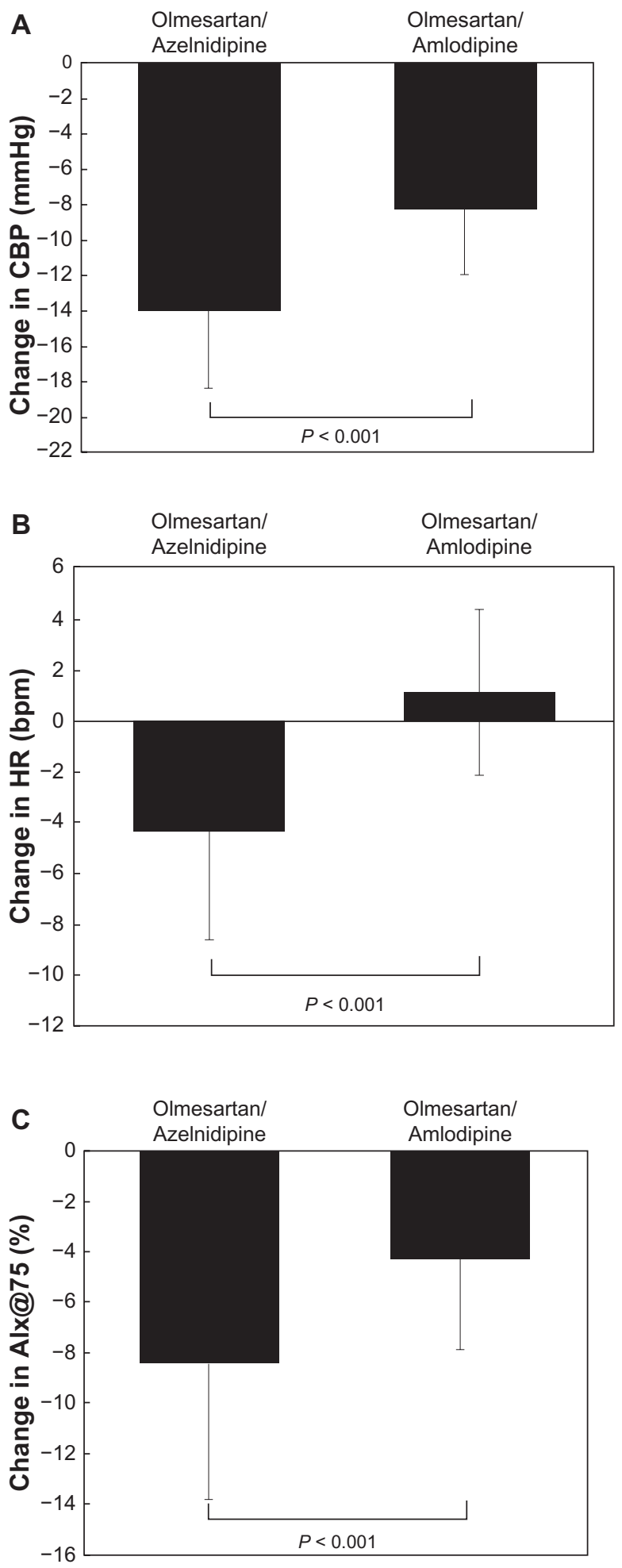

Figure 2 (Continued) 

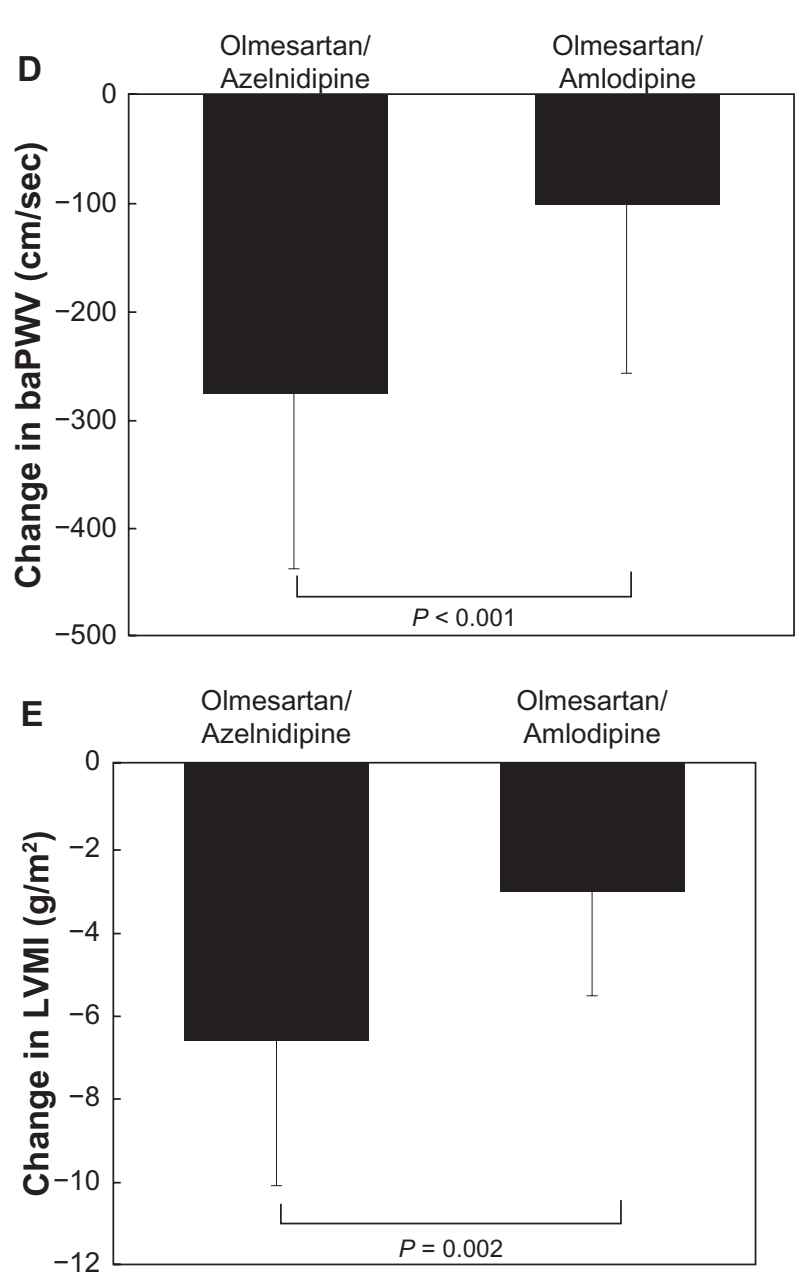

Figure 2 Changes in central blood pressure (CBP) (A), heart rate (HR, beats per minute $[\mathrm{bpm}])(\mathbf{B})$, normalized augmentation index (Alx@75) (C), baPWV (D), and left ventricular mass index (LVMI) (E) from baseline to week 24.

in the olmesartan/azelnidipine group than in the olmesartan/ amlodipine group $(-275.9 \pm 161.2 \mathrm{vs}-101.6 \pm 155.6 \mathrm{~cm} /$ second, respectively, $P<0.001$; Figure 2D).

\section{Changes in LVMI}

LVMI decreased significantly in both treatment groups between baseline and endpoint (both, $P<0.001$ ). Notably, the magnitude of the decrease in LVMI was significantly greater in the olmesartan/azelnidipine group than in the olmesartan/amlodipine group (-6.6 $\pm 3.4 \mathrm{vs}-3.0 \pm 2.5 \mathrm{~g} / \mathrm{m}^{2}$, respectively, $P=0.002$; Figure $2 \mathrm{E}$ ). We also detected a strong correlation between the change in CBP and the change in LVMI $\left(R^{2}=0.6262, P<0.0001\right.$; Figure 3$)$.

\section{Adverse events}

All of the patients who entered the randomized phase of the study completed the study without experiencing any serious adverse events or drug-related adverse events.

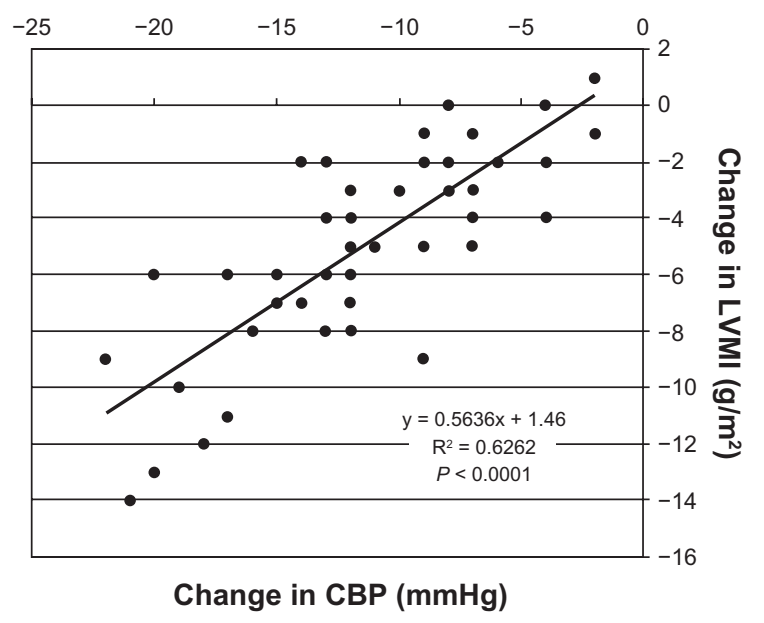

Figure 3 Changes in central blood pressure (CBP) and left ventricular mass index (LVMI) between baseline and endpoint.

\section{Discussion}

In this study, we found that, during 24 weeks of combination therapy, olmesartan/azelnidipine elicited significantly greater decreases in CBP than olmesartan/amlodipine, even though the decrease in brachial BP was similar with both treatments. In addition, AIx@75, baPWV, and HR all showed significantly greater decreases with olmesartan/azelnidipine than with olmesartan/amlodipine.

Different hemodynamic effects of the two regimens may explain why the decrease in CBP was greater with olmesartan/ azelnidipine than with olmesartan/amlodipine, a concept supported by the greater decrease in AIx@75 with olmesartan/ azelnidipine than with olmesartan/amlodipine. AI is a measure of the contribution of wave reflection to the aortic waveform and depends on the pulse wave velocity, and magnitude and site of the reflected pressure wave, and thus provides a composite measure of large artery (systemic) stiffness. ${ }^{16}$ Increases in CBP and the AIx@75 are influenced by pressure waves that are reflected back toward the heart from branch points throughout the arterial tree. PWV is also a measure of arterial stiffness. When arteries become sclerosed, PWV is elevated, promoting an acceleration of the waves reflected back. Renin-angiotensin system (RAS) inhibitors and CCBs act directly on peripheral sites, alter the arterial smooth muscle, and thus reduce pressure wave reflection. ${ }^{2,17-21}$ In fact, RAS inhibitors and CCBs have been shown to decrease $\mathrm{CBP}$ by reducing pressure wave reflection. ${ }^{22}$ Compared with amlodipine, azelnidipine may elicit greater reductions in CBP by inducing greater decreases in pressure wave reflection. Thus, the main mechanism responsible for CBP lowering associated with olmesartan/ azelnidipine may involve reductions in the magnitude of and potential delays in pressure wave reflection. 
Another mechanism that may explain the decrease in CBP in this study is that olmesartan/azelnidipine decreased the HR whereas olmesartan/amlodipine did not. These results are consistent with previous reports in which azelnidipine decreased clinic-measured HR and 24-hour HR, whereas amlodipine increased both of these parameters, despite similar BP reductions. ${ }^{8,23}$ It was also reported that, after switching from amlodipine, azelnidipine exhibited hypotensive effects compared with amlodipine, and significantly decreased HR and the total number of extrasystole phases. ${ }^{24}$ Noradrenaline levels and the LF/HF ratio were significantly decreased, and the washout rate was significantly reduced on 123I-MIBG myocardial scintigraphy. These findings suggest that azelnidipine inhibits the enhancement of sympathetic nervous activity. ${ }^{24}$ These differences in the effects of azelnidipine and amlodipine can also be explained by the finding that azelnidipine exerts stronger inhibition of sympathetic activity in the vasodilation-induced baroreceptor reflex than does amlodipine. ${ }^{7}$

Experimental studies have also shown that azelnidipine dose-dependently reduces HR. ${ }^{25}$ In another study, azelnidipine suppressed cardiac hypertrophy, fibrosis, NADPH oxidase, and superoxide levels in stroke-prone spontaneously hypertensive rats more potently than amlodipine, and was associated with lower HR than amlodipine. ${ }^{26}$ Azelnidipine also caused a greater reduction than amlodipine in the beat rate of the sinus node/atrial preparation of these rats. ${ }^{26}$ Based on these findings, olmesartan/azelnidipine may reduce CBP via the HR-lowering effect of azelnidipine.

In general, AI and CBP increase with HR lowering. In this study, however, both parameters decreased despite HR lowering. This result is consistent with a previous report showing that olmesartan/azelnidipine significantly decreased HR, AI, CBP, and Aortic PWV (AoPWV) by more than olmesartan/hydrochlorothiazide. ${ }^{5}$ The HR-lowering effect of azelnidipine through its sympathetic inhibition may be at least partly responsible for the greater effect of the olmesartan/azelnidipine combination on AoPWV. ${ }^{5}$ We measured baPWV rather than AoPWV, but it has been reported that baPWV correlates well with AoPWV. ${ }^{27}$

In this study, we also found that the decrease in LVMI was significantly greater with olmesartan/azelnidipine than with olmesartan/amlodipine. The strong correlation between change in LVMI and change in CBP (Figure 3), suggests that the greater reduction in LVMI in the olmesartan/azelnidipine group may be related to the greater reduction of CBP in that group. In hypertension the pressure reflection wave during systole augments central ascending aortic BP. ${ }^{17,28,29}$ As a result, the LV must provide greater force during ejection to overcome the augmented pressure. ${ }^{30,31} \mathrm{AI}, \mathrm{CBP}$ and wasted LV effort were positively associated with LV hypertrophy in untreated hypertensive patients. ${ }^{32}$ It was also reported that an increase in the ascending aortic AI caused by a reflected wave may be involved in the formation of LV hypertrophy. ${ }^{33}$ Moreover, in the REASON study, the greater change in LV mass was linked to CBP but not brachial BP. ${ }^{34}$ Thus, LVMI lowering associated with olmesartan/azelnidipine may have been due to reductions in CBP and AI.

It has been reported that lowering BP with the additional use of azelnidipine is associated with improvements in LV diastolic performance (ie, an increase in the e' velocity), a reduction in $\mathrm{LV}$ filling pressure (a decrease in the E/e' ratio) and a decrease in the brain natriuretic peptide level in patients with hypertension and a preserved systolic function. ${ }^{35}$ Among patients in whom amlodipine was switched to azelnidipine, $\mathrm{BP}$ and $\mathrm{HR}$ decreased significantly, and these reductions were associated with an increase in the e' velocity. ${ }^{35}$ Therefore, regression of LVMI may be related to the improvements in LV diastolic function elicited by azelnidipine.

CBP, AI, and LV hypertrophy are independent predictors of cardiovascular morbidity in patients with hypertension. , 36,37 $^{2}$ Epidemiological studies have shown that an increased HR is associated with a higher incidence of cardiovascular diseases and a worse prognosis. ${ }^{38,39}$ Tachycardia caused by reflex activation of the sympathetic nervous system is a major adverse effect of CCB therapy. However, unlike amlodipine, azelnidipine has been shown to inhibit sympathetic activity, ${ }^{40}$ suggesting that tachycardia is unlikely to occur with azelnidipine. Indeed, we found that the HR decreased with azelnidipine, but not with amlodipine.

Some limitations of this study should be discussed. First, this was not a double-blind randomized trial, meaning the possibility of a significant bias cannot be excluded. However, measurements were performed by a single clinical investigator who was blinded to the treatment allocation; therefore, there was no bias in the measurement and evaluation of laboratory data. Second, the study period was relatively short and the sample size was small; longer and larger studies are necessary to evaluate the long-term effects of these combination therapies on arterial structure. Third, the dose of azelnidipine used might also have influenced the results. However, we used the standard clinical dose of azelnidipine (ie, $16 \mathrm{mg} /$ day), a dose that achieved similar reductions in BP to that achieved by a standard dose of amlodipine (ie, $5 \mathrm{mg}$ /day). Finally, the CBP results are based solely on radial tonometry data. Radial tonometry is comfortable for patients 
and easy to use in a clinical setting, but it is subject to interobserver variability and the results may not be an accurate reflection of actual pressures. Therefore, future studies should validate these results using direct pressure measurements.

\section{Conclusion}

In conclusion, the AORTA study revealed that olmesartan in combination with azelnidipine elicited greater reductions in CBP and LVMI compared with olmesartan in combination with amlodipine, even though the reduction in brachial BP was similar with both regimens. CBP is an independent predictor of cardiovascular morbidity in hypertensive patients. Therefore, the superior effects of olmesartan/azelnidipine therapy on central hemodynamics may be associated with more favorable cardiovascular outcomes than with olmesartan/amlodipine. Such differential effects may be important for cardiovascular risk reduction and warrant large-scale, long-term clinical trials to confirm this hypothesis.

\section{Acknowledgment}

The authors wish to acknowledge Noriko Mune for technical support. No grants were used to fund this study.

\section{Disclosure}

The authors declare no conflicts of interest in this work.

\section{References}

1. Ogihara T, Kikuchi K, Matsuoka H, et al. The Japanese Society of Hypertension Guidelines for the Management of Hypertension (JSH 2009). Hypertens Res. 2009;32:3-107. [Erratum: Hypertens Res. 2009;32:318].

2. Williams B, Lacy PS, Thom SM, et al. Differential impact of blood pressure-lowering drugs on central aortic pressure and clinical outcomes: principal results of the Conduct Artery Function Evaluation (CAFÉ) study. Circulation. 2006;113:1213-1225.

3. Roman MJ, Devereux RB, Kizer JR, et al. Central pressure more strongly relates to vascular disease and outcome than does brachial pressure: the Strong Heart Study. Hypertension. 2007;50:197-203.

4. Miyashita H, Aizawa A, Hashimoto J, et al. Cross-sectional characterization of all classes of antihypertensives in terms of central blood pressure in Japanese hypertensive patients. Am J Hypertens. 2010; 23:260-268.

5. Matsui Y, Eguchi K, O’Rourke MF, et al. Differential effects between a calcium channel blocker and a diuretic when used in combination with angiotensin II receptor blocker on central aortic pressure in hypertensive patients. Hypertension. 2009;54:716-723.

6. Kondo T, Goto R, Sonoda K, et al. Plasma renin activity and aldosterone concentration are not altered by the novel calcium channel antagonist, azelnidipine, in hypertensive patients. Intern Med. 2010;49:637-643.

7. Eguchi K, Tomizawa H, Ishikawa J, et al. Effects of new calcium channel blocker, azelnidipine, and amlodipine on baroreflex sensitivity and ambulatory blood pressure. J Cardiovasc Pharmacol. 2007;49: 394-400.

8. Nakamura T, Sugaya T, Kawagoe Y, et al. Azelnidipine reduces urinary protein excretion and urinary liver-type fatty acid binding protein in patients with hypertensive chronic kidney disease. Am J Med Sci. 2007; 333:321-326.
9. Melenovsky V, Borlaug BA, Fetics B, Kessler K, Shively L, Kass DA. Estimation of central pressure augmentation using automated radial artery tonometry. J Hypertens. 2007;25:1403-1409.

10. Takazawa K, Kobayashi H, Shindo N, Tanaka N, Yamashina A. Relationship between radial and central arterial pulse wave and evaluation of central aortic pressure using the radial arterial pulse wave. Hypertens Res. 2007;30:219-228.

11. Richardson CJ, Maki-Petaja KM, McDonnell BJ, Hickson SS, Wilkinson IB, McEniery CM. Comparison of estimates of central systolic blood pressure and peripheral augmentation index obtained from the Omron HEM-9000 AI and SphygmoCor systems. Artery Res. 2009;3:24-31

12. Hickson SS, Butlin M, Mir FA, et al. The accuracy of central SBP determined from the second systolic peak of the peripheral pressure waveform. J Hypertens. 2009;27:1784-1788.

13. Wilkinson IB, Mohammad NH, Tyrrell S, et al. Heart rate dependency of pulse pressure amplification and arterial stiffness. Am J Hypertens. 2002;15:24-30.

14. Takami T. Evaluation of arterial stiffness in morning hypertension under high-dose valsartan compared to valsartan plus low-dose diuretic. Hypertens Res. 2009;32:1086-1090.

15. Devereux RB, Palmieri V, Sharpe N, et al. Effects of once-daily angiotensin-converting enzyme inhibition and calcium channel blockade-based antihypertensive treatment regimens on left ventricular hypertrophy and diastolic filling in hypertension: the prospective randomized enalapril study evaluating regression of ventricular enlargement (PRESERVE) trial. Circulation. 2001;104:1248-1254.

16. Safar ME, London GM. Therapeutic studies and arterial stiffness in hypertension: recommendations of the European Society of Hypertension. The Clinical Committee of Arterial Structure and Function. Working Group on Vascular Structure and Function of the European Society of Hypertension. J Hypertens. 2000;18:1527-1535.

17. Asmar RG, O'Rourke MF, Safar ME; on behalf of the REASON Project Investigators. Mechanism(s) of selective systolic blood pressure reduction after a low-dose combination of perindopril/indapamide in hypertensive subjects: comparison with atenolol. J Am Coll Cardiol. 2004;43:92-99.

18. Stokes GS, Barin WS, Gilfillan KL. Effects of isosorbide mononitrate and AII inhibition on pulse wave reflection in hypertension. Hypertension. 2003;41:297-301.

19. Stewart AD, Millasseau SC, Kearney MT, Ritter JM, Chowienczyk PJ. Effects of inhibition of basal nitric oxide synthesis on carotid-femoral pulse wave velocity and augmentation index in humans. Hypertension. 2003;42:915-918.

20. Safar ME. Mechanisms of systolic blood pressure reduction and drug therapy in hypertension. Hypertension. 2007;50:167-171.

21. Asmar RG, London GM, O'Rourke MF, Safar ME. Improvement in blood pressure, arterial stiffness and wave reflections with a very-low-dose perindopril/indapamide combination in hypertensive patient: a comparison with atenolol. Hypertension. 2001;38: 922-926.

22. Agabiti-Rosei E, Mancia G, O’Rourke MF, et al. Central blood pressure measurements and antihypertensive therapy: a consensus document. Hypertension. 2007;50:154-160.

23. Kuramoto K, Ichikawa S, Hirai A, Kanada S, Nakachi T, Ogihara T. Azelnidipine and amlodipine: a comparison of their pharmacokinetics and effects on ambulatory blood pressure. Hypertens Res. 2003; 26:201-208

24. Nada T, Nomura M, Koshiba K, Kawano T, Mikawa J, Ito S. Clinical study with azelnidipine in patients with essential hypertension. Antiarteriosclerotic and cardiac hypertrophy-inhibitory effects and influence on autonomic nervous activity. Arzneimittelforschung. 2007;57:698-670.

25. Fujisawa M, Yorikane R, Chiba S, Koike H. Chronotropic effects of azelnidipine, a slow- and long-acting dihydropyridine-type calcium channel blocker, in anesthetized dogs: a comparison with amlodipine. J Cardiovasc Pharmacol. 2009;53:325-332. 
26. Yamamoto E, Lai ZF, Yamashita T, et al. Enhancement of cardiac oxidative stress by tachycardia and its critical role in cardiac hypertrophy and fibrosis. J Hypertens. 2006;24:2057-2069.

27. Yamashina A, Tomiyama H, Takeda K, et al. Validity, reproducibility, and clinical significance of noninvasive brachial-ankle pulse wave velocity measurement. Hypertens Res. 2002;25:359-364.

28. Safar ME, Boudier HS. Vascular development, pulse pressure, and the mechanisms of hypertension. Hypertension. 2005;46:205-209.

29. Safar ME. Systolic hypertension in the elderly: arterial wall mechanical properties and the renin-angiotensin-aldosterone system. J Hypertens. 2005;23:673-681.

30. Nichols WW. Clinical measurement of arterial stiffness obtained from noninvasive pressure waveforms. Am J Hypertens. 2005;18: $3 \mathrm{~S}-10 \mathrm{~S}$.

31. Nichols WW, Estrada JC, Braith RW, Owens K, Conti CR. Enhanced external counterpulsation treatment improves arterial wall properties and wave reflection characteristics in patients with refractory angina. J Am Coll Cardiol. 2006;48:1208-1214.

32. Hashimoto J, Nichols WW, O'Rourke MF, Imai Y. Association between wasted pressure effort and left ventricular hypertrophy in hypertension: influence of arterial wave reflection. Am J Hypertens. 2008;21:329-333.

33. Iketani T, Iketani Y, Takazawa K, Yamashina A. The influence of the peripheral reflection wave on left ventricular hypertrophy in patients with essential hypertension. Hypertens Res. 2000;23:451-458.
34. de Luca N, Asmar RG, London GM, O'Rourke MF, Safar ME. REASON Project Investigators. Selective reduction of cardiac mass and central blood pressure on low-dose combination perindopril/indapamide in hypertensive subjects. J Hypertens. 2004;22:1623-1630.

35. Ito $\mathrm{H}$, Ishii $\mathrm{K}$, Iwakura $\mathrm{K}$. Impact of azelnidipine treatment on left ventricular diastolic performance in patients with hypertension and mild diastolic dysfunction: multi-center study with echocardiography. Hypertens Res. 2009;32:895-900.

36. Hashimoto J, Imai Y, O'Rourke MF. Indices of pulse wave analysis are better predictors of left ventricular mass reduction than cuff pressure. Am J Hypertens. 2007;20:378-338.

37. Iwashima Y, Horio T, Kamide K, Rakugi H, Ogihara T, Kawano Y. Uric acid, left ventricular mass index, and risk of cardiovascular disease in essential hypertension. Hypertension. 2006;47:195-202.

38. Gillman MW, Kannel WB, Belanger A, D’Agostino RB. Influence of heart rate on mortality among persons with hypertension: the Framingham Study. Am Heart J. 1993;125:1148-1154.

39. Seccareccia F, Pannozzo F, Dima F, et al; Malattie Cardiovascolari Aterosclerotiche Istituto Superiore di Sanita Project. Heart rate as a predictor of mortality: the MATISS project. Am J Public Health. 2001; 91:1258-1263.

40. Shokoji T, Fujisawa Y, Kiyomoto H, et al. Effects of a new calcium channel blocker, azelnidipine, on systemic hemodynamics and renal sympathetic nerve activity in spontaneously hypertensive rats. Hypertens Res. 2005;28:1017-1023.
Vascular Health and Risk Management

\section{Publish your work in this journal}

Vascular Health and Risk Management is an international, peerreviewed journal of therapeutics and risk management, focusing on concise rapid reporting of clinical studies on the processes involved in the maintenance of vascular health; the monitoring, prevention and treatment of vascular disease and its sequelae; and the involvement of

\section{Dovepress}

metabolic disorders, particularly diabetes. This journal is indexed on PubMed Central and MedLine. The manuscript management system is completely online and includes a very quick and fair peer-review system, which is all easy to use. Visit http://www.dovepress.com/ testimonials.php to read real quotes from published authors. 